\title{
情境教学法在高中英语教学中的应用探究
}

\author{
陈珞 \\ 重庆市涪陵第十七中学校 \\ DOI:10.32629/er.v3i7.3008
}

\begin{abstract}
[摘 要] 新课改的有效推进, 随之涌现出许多新型教学模式, 情境教学法便是其中之一。这种教学法要 求教师在教学过程中有目的地引入或创设生动形象的场景, 以使学生深入情境中体验和学习, 从而帮助 学生更好的理解教学内容。本文针对情境教学法在高中英语教学中的应用进行探讨, 并提出相应的教学 策略, 旨在提高高中英语课堂教学的效果, 提升学生英语水平和应用能力, 促进学生的全面发展。
\end{abstract}

[关键词] 情境教学法; 高中英语教学; 应用

英语新课程标准中明确指出, 教师 要关注学生情感体验的优化和学习兴趣 的激发, 将学生从被动学习的状态中解 放出来, 调整教学方法和策略, 以促进学 生的自主、合作和探究学习, 实现教学实 效的提升和学生知识、能力的全面发展。 所以, 作为高中英语教师, 我们必须要顺 应改革发展的趋势, 摆脱传统观念的束 缚, 遵循新课标的理念和要求, 积极推进 教学方法的革新, 构建情境化的英语教 学模式, 切实提升高中英语教学的效能。

\section{1 情境教学法的特征分析}

1. 1 直观性

在高中英语教学中采用情境教学法, 就是借助语言描述、外界事物等方式, 让学生能融入到英语课堂中, 让学生能 更好地掌握英语知识, 提高学生的阅读 能力和分析能力。在英语情境中, 可以将 原本抽象的英语知识转变成具体的情境 活动, 可以明确学生的学习方向, 因而情 景教学法具有直观性。

\section{2 启发性}

启发性指的是, 当学生能处于一个 良好的环境后, 可以在环境、教师的引导 下, 向着既定的目标前进和发展。在高中 英语的教学过程中, 教师需要采用多样 化的情境教学模式, 让学生可以在情境 中更好地思考, 锻炼学生的想象能力, 让 学生能更好地掌握英语知识。因此, 情景 教学法具有较强的启发性。

\section{3寓教于乐性}

高中英语教师在实际教学过程中, 需要重视学生学习兴趣的培养。在英语 情境中, 可以通过肢体表演、角色设定、 视频动画等方式, 促使整个英语课堂变 得更具有娱乐性, 学生可以在这样的氛 围中更好地学习和阅读。因此, 情境教学 法具有寓教于乐性。

\section{2 高中英语课堂开展情境化教} 学的必要性

\section{1 创建锻炼语言能力的情境}

英语是高中教学中最重要的学科之 一, 而且作为一门语言学科, 就更加需要 学生能够熟练掌握其表达方式。但是由 于英语不是作为母语出现的, 所以学生 在学习过程中往往会遇到诸多难题, 这 时候教师就可以采用情境教学法来应对 这些问题, 结合当下的教学内容, 为学生 创立合适的语言情境, 使得学生能够敢 于开口, 最大限度地激发起学生对于英 语学习的兴趣。合理的语境能够帮助学 生练习口语, 巩固课堂中学习过的知识 点, 还能够锻炼学生的英语听力, 提升其 自主学习的能力。

\section{2 建立语言实践的情境}

教师可以通过情境化教学为学生提 供锻炼口语的场景, 将实际生活与教学 内容相结合, 将高中英语教学与实际相 关联, 以此来实现英语交际的教学目的, 提高学生的英语实践能力。基于生活的 英语情境化教学能够使学生进一步了解 到英语在日常生活的某些确切用法, 落
实英语口语教学的最终目的, 使学生能 够在交流过程中正确地使用英语。

\section{3 巩固书本理论知识}

基于信息技术的飞速发展, 我国绝 大部分高中已经引进了多媒体教学, 以 现代化手段作为辅助, 摒弃传统教学的 弊端, 有效实现课堂教学大容量、高效 率。教师在高中英语教学过程中, 可以以 信息技术手段为基本, 为学生创建相应 的教学情境, 深化学生对于书本理论知 识的理解。情境教学法能够将抽象的知 识通过实际表现出来, 例如视频、图片、 音频等。这种教学方法能够使学生直观 形象地了解到英语与汉语之间存在的不 同, 避免在学习过程中受到汉语语言特 征的影响, 为学生提供多方面、多角度的 学习途径。

\section{3 情境教学法在高中英语课堂 中的实际应用}

3. 1 借助语境完成词汇记忆

对于每一个阶段的学生来说, 词汇 学习既是基础性内容, 也是核心内容。只 有在掌握扎实词汇知识的基础上,才能 对相对应的句型和语法知识有更加直观 的了解和掌握。而在词汇学习的过程中, 长期按照单一的死记硬背模式, 不仅会 造成学生记忆效率低下, 还会引发一系 列问题, 比如学生对词汇记忆出现混淆 以及记忆错误的问题。转变传统的词汇 记忆形式, 让学生以新型的方法完成词 汇学习是提高学生学习热情以及词汇学 
习效率的关键, 其中借助语境是一种非 常有效的形式。在结合具体语境学习词 汇时, 学生可以在对词汇使用的具体语 境产生了解的同时, 对具体词汇不同方 面的含义有更加深刻的印象。在单一的 词汇记忆过程中, 学生需要死记硬背, 也 很容易出现混淆, 而在结合具体语境进 行学习时, 学生可以通过整个句子的具 体内容, 推断出词汇的含义。在组织学生 进行词汇学习时, 创设语言情境是非常 有效的方法, 反馈到高中英语具体教学 中, 相关语境的创设可以借助多媒体设 施来完成, 教师可以通过制作精致课件 的形式, 让学生了解具体词汇在不同语 境中的相关应用, 进而了解其不同方面 的内涵。

\section{2 借助情境理解语言运用}

在词汇学习的基础上, 掌握语法知 识对于学生英语水平提升来说也是同样 重要的。语法是构成英语的骨干, 只有在 了解语法知识的前提下, 学生才能够准 确利用英语表达出自己想要表达的内容, 熟悉语言运用, 提高自己的语言表达能 力, 对学生来说是非常重要的。一方面, 在完成英语考试中的写作时, 学生需要 在有限的时间内使自己的写作更加高效, 在准确表达出题目要求内容的前提下使 自己的文章更加出彩。另一方面, 结合核 心素养要求下的高中英语教学, 学生应 该掌握必要的英语口语表达能力, 能够 完成日常英语对话, 掌握语法知识, 才能 保证对方在与自己完成对话的过程中准
确理解自己表达的内容。在教学具体知 识时, 进行情境创设是非常有效的方法, 能够让学生换一种方式学习语法知识, 同时在参与具体的情境中, 学生的语言 表达能力也会提高。例如在教学 “短语 动词的被动语态”这一知识点时, 短语动 词是一个整体, 不可丢掉后面的介词或 副词。为了让学生有更加准确的理解, 教师可以创建语言情境, 通过汉语引导 的形式, 让学生进行对话的构建, 教师可 以让学生根据自己的知识储备翻译 “我 妹妹由奶奶照顾”, 最后通过不断修正得 出 “My sister will be taken care of by Grandma” 这一正确的语言表述。

\section{3 丰富情境创设形式}

情境教学法适用于各个阶段学生的 英语学习, 而在高中阶段的情境创设过 程中, 其主要目标不仅侧重于提升学生 的学习兴趣, 而是让学生对英语知识有 更加深入地了解。结合高中阶段学生的 英语学习情况, 为了有效激发英语基础 较差学生的学习热情, 同时鼓励基础较 好的学生不断提升自己, 教师应该丰富 情境创设的基本形式, 使英语课堂更具 有活力。在不同内容的教学过程中, 情境 创设方法的应用可以呈现出多样化的特 征。在教学文本类内容时, 教师可以组织 学生进行情境创设, 通过播放视频的形 式来了解文章中所阐述的具体内容, 在 观看视频的过程中, 学生的投入度更高, 而在讲解语法类等固定知识的过程中, 通过对话情境的模拟也可以让学生有更
加深刻的印象, 调动学生学习积极性。转 变对语法知识这一相对枯燥内容的学习 态度。例如在教学 “Festivals around the world” 这一单元内容时, 为了让学 生对文章内容有较高的学习热情, 进而 产生更高的投入, 了解文章中表达的具 体内容的同时, 对重点词汇和语法知识 有深入了解, 教师可以通过播放视频的 形式来吸引学生, 创设与 “Festivals around the world” 相关地具体情境。 在选择视频时, 教师可以搜集介绍欧美、 亚太等不同地区、不同风格以及特色节 日的视频。在观看这一视频的过程中, 学生会对其中的几个节日产生比较浓厚 的兴趣, 进而在学习相关文章的过程中 有较高的热情。

\section{4 结语}

在高中英语的教学过程中, 要想让 学生对英语知识的学习保持充足的兴趣, 那么就需要教师能结合教学内容创设多 样化的教学情境, 如生活情境、问题情境 等。这样, 学生才能更好地融入到英语课 堂中, 实现个人能力和素养的同步提升。

\section{[参考文献]}

[1]李向红.情境教学法在高中 英语语法教学中的应用 [J]. 英语教 师,2019,19(17):150-152.

[2]叶晶伟.谈情境教学在高中英语 教学中的实践 [J].才智,2019,(33):46.

[3] 楼蕾.情境教学法在高中英语语法 教学中的应用[J].现代教学,2017,(7):61-62. 